\title{
Chromatic Pupillometry Findings in Alzheimer's Disease
}

\author{
Martina Romagnoli', Michelangelo Stanzani Maserati ', Maddalena De Matteis', \\ Sabina Capellari ${ }^{1,2}$, Michele Carbonelli ${ }^{1}$, Giulia Amore ${ }^{2}$, Gaetano Cantalupo ${ }^{3}$, \\ Corrado Zenesini', Rocco Liguori ${ }^{1,2}$, Alfredo A. Sadun ${ }^{4}$, Valerio Carelli ${ }^{1,2}$, Jason C. Park ${ }^{5}$ \\ and Chiara La Morgia ${ }^{1,2 *}$
}

\begin{abstract}
${ }^{1}$ IRCCS Istituto Delle Scienze Neurologiche di Bologna, UOC Clinica Neurologica, Ospedale Bellaria, Bologna, Italy, ${ }^{2}$ Department of Biomedical and Neuromotor Sciences, University of Bologna, Bologna, Italy, ${ }^{3}$ Division of Child Neuropsychiatry, Department of Surgical Sciences, Dentistry, Gynaecology and Paediatrics, University of Verona, Verona, Italy, ${ }^{4}$ Department of Ophthalmology, Doheny Eye Institute, University of California, Los Angeles, Los Angeles, CA, United States, ${ }^{5}$ Department of Ophthalmology and Visual Sciences, University of Illinois at Chicago, Chicago, IL, United States
\end{abstract}

\section{OPEN ACCESS}

Edited by:

Silvia Di Angelantonio,

Sapienza University of Rome, Italy

Reviewed by:

Kwoon Y. Wong,

University of Michigan, United States

Jordan M. Renna,

The University of Akron, United States

*Correspondence:

Chiara La Morgia

chiara.lamorgia@unibo.it;

chiaralamorgia@gmail.com

Specialty section:

This article was submitted to

Neurodegeneration,

a section of the journal

Frontiers in Neuroscience

Received: 09 May 2020

Accepted: 02 July 2020

Published: 11 August 2020

Citation:

Romagnoli M, Stanzani

Maserati M, De Matteis M,

Capellari S, Carbonelli M, Amore G,

Cantalupo G, Zenesini C, Liguori R,

Sadun AA, Carelli V, Park JC and

La Morgia C (2020) Chromatic

Pupillometry Findings in Alzheimer's

Disease. Front. Neurosci. 14:780.

doi: 10.3389/fnins.2020.00780
Intrinsically photosensitive melanopsin retinal ganglion cells (mRGCs) are crucial for nonimage forming functions of the eye, including the photoentrainment of circadian rhythms and the regulation of the pupillary light reflex (PLR). Chromatic pupillometry, using light stimuli at different wavelengths, makes possible the isolation of the contribution of rods, cones, and mRGCs to the PLR. In particular, post-illumination pupil response (PIPR) is the most reliable pupil metric of mRGC function. We have previously described, in post-mortem investigations of $A D$ retinas, a loss of mRGCs, and in the remaining mRGCs, we demonstrated extensive morphological abnormalities. We noted dendrite varicosities, patchy distribution of melanopsin, and reduced dendrite arborization. In this study, we evaluated, with chromatic pupillometry, the PLR in a cohort of mildmoderate $A D$ patients compared to controls. $A D$ and controls also underwent an extensive ophthalmological evaluation. In our AD cohort, PIPR did not significantly differ from controls, even though we observed a higher variability in the AD group and 5/26 showed PIPR values outside the 2 SD from the control mean values. Moreover, we found a significant difference between $A D$ and controls in terms of rod-mediated transient PLR amplitude. These results suggest that in the early stage of AD there are PLR abnormalities that may reflect a pathology affecting $\mathrm{mRGC}$ dendrites before involving the mRGC cell body. Further studies, including AD cases with more severe and longer disease duration, are needed to further explore this hypothesis.

Keywords: chromatic pupillometry, Alzheimer's disease, melanopsin retinal ganglion cells, pupillary light reflex, post-illumination pupil response, pupil

\section{INTRODUCTION}

Melanopsin retinal ganglion cells (mRGCs) are intrinsically photosensitive RGCs because of the expression of the photopigment melanopsin (Berson et al., 2002; Hannibal et al., 2002; Hattar et al., 2002). These cells contribute to non-image forming functions of the eye including circadian photoentrainment [projecting via the retino-hypothalamic tract (RHT) to the suprachiasmatic nucleus (SCN) of the hypothalamus] and regulation of the pupillary light reflex (PLR) [via projections to the olivary pretectal nucleus (OPN)] (Sadun et al., 1984; Hannibal et al., 2004, 2014; Baver et al., 2008; Chen et al., 2011; Li and Schmidt, 2018). 
Neurodegenerative disorders, including Alzheimer's disease $(A D)$, are characterized by prominent circadian and sleep dysfunction even in the early phase of the disease (Uddin et al., 2020). Melanopsin retinal ganglion cell loss demonstrated in post-mortem $\mathrm{AD}$ retinas may contribute to the circadian and sleep problems documented in these patients (La Morgia et al., 2016, 2017).

Amyloid plaques have been detected in $\mathrm{AD}$ retinas (Koronyo et al., 2017) and amyloid pathology can also affect mRGCs, suggesting a specific mechanism of neurodegeneration independent from the aging process (La Morgia et al., 2016). Moreover, extensive morphological abnormalities with dendrite varicosities, patchy distribution of melanopsin, and reduced dendrite arborization were noted in remaining mRGCs of $\mathrm{AD}$ retinas (La Morgia et al., 2016).

The function of mRGCs is, however, difficult to explore in vivo, since these cells represent a small subgroup (about 1\%) of the regular RGCs, and also mRGCs receive some input from rods and cones (Hannibal et al., 2017). Chromatic pupillometry protocols have been developed to isolate the contribution of mRGCs to the PLR and to assess in vivo the function of mRGCs (Kardon et al., 2011; Park et al., 2011; La Morgia et al., 2018). These protocols are based on light stimuli at different wavelengths and with light adaptation conditions aimed at isolating the contribution of single photoreceptors, taking into account that mRGCs are maximally sensitive to blue light at $480 \mathrm{~nm}$ (Berson et al., 2002). It has been shown that the post-illumination pupil response (PIPR) is the most reliable pupil metric of mRGC function (Adhikari et al., 2015b).

Previous studies investigated the presence of pupil abnormalities in $\mathrm{AD}$ patients but they used different visual stimuli, heterogeneous protocols, and results were not consistent (Chougule et al., 2019). Pre-symptomatic cases (Oh et al., 2019; Van Stavern et al., 2019) and, recently, early AD cases (Kawasaki et al., 2020) were evaluated with chromatic pupillometry to isolate the mRGC contribution.

The present study was designed to evaluate the PLR, and in particular the mRGC-mediated contribution, in $\mathrm{AD}$. We here report chromatic pupillometry findings using a previously published protocol (Park et al., 2017) in a cohort of 26 mildmoderate $\mathrm{AD}$ patients and 26 controls for which a detailed neuro-ophthalmological evaluation has been performed.

\section{METHODS}

\section{Study Participants}

This is a cross-sectional study and follows the STROBE guidelines (von Elm et al., 2007). We included AD patients and healthy controls, evaluated between June 2017 and February 2020 at the IRCCS Institute of Neurological Sciences of Bologna. All subjects gave written informed consent for the prospective collection of clinical data, data analyses, and publication. The study was conducted in agreement with the Declaration of Helsinki and approved by the local ethical committee (EC Interaziendale Bologna-Imola \#16032) and within the framework of the research project supported by the Italian Ministry of Health, GR-2013-02358026 to CLM. We included patients with a diagnosis of $\mathrm{AD}$ according to Dubois criteria (Dubois et al., 2014) and National Institute of Neurological and Communication Disorders-Alzheimer's Disease and Related Disorders Association criteria (NINCDSADRDA) (McKhann et al., 1984) at mild-moderate stage [MiniMental State Examination (MMSE) score between 11 and 26] (Folstein et al., 1975).

The absence of cognitive dysfunction was ascertained in the control group.

Exclusion criteria for both control and $\mathrm{AD}$ groups were: spherical or cylindrical refractive errors more than 3 or 2 diopters, respectively; presence of posterior pole pathology including agerelated macular degeneration and known optic neuropathies (including open-angle glaucoma); ocular pressure more than $20 \mathrm{mmHg}$; severe lens opacity and/or retinal detachment and/or vascular retinal pathology (including diabetic retinopathy); history of ophthalmologic surgery, except for uncomplicated cataract surgery, performed at least 6 months previously; shiftworkers in the last year; travels through more than one time zone during the last 3 months.

All study participants completed self-administered questionnaires including Epworth Sleepiness Scale (ESS), Pittsburgh Sleep Quality Index (PSQI), Berlin questionnaire, and Beck Anxiety (BAI) and Depression Inventory (BDI) (Beck et al., 1961, 1988) to evaluate the possible occurrence of sleep disturbances. For the control group, exclusion criteria included also the presence of the following abnormal scores at sleep and mood questionnaires: excessive daytime sleepiness as assessed by the ESS (Vignatelli et al., 2003); presence of sleep disturbances as determined by the PSQI (Buysse et al., 1989); abnormal scores on the BAI (Beck et al., 1988) and BDI (Beck et al., 1961) tests.

All subjects underwent an extensive neuro-ophthalmological evaluation including visual acuity testing, tonometry, fundus examination, Ishihara color vision test, and OCT examination. OCT examination was performed using SS (Swept-Source)OCT with the deep range imaging (DRI) Triton OCT (Topcon, Japan) using the 3DWide $12 \times 9 \mathrm{~mm}$ scan protocol including segmentation analysis. We evaluated the average and 4 individual quadrants (temporal, superior, nasal, and inferior) peripapillary Retinal Nerve Fiber Layer (pRNFL) thickness, and the average and 6 individual macular sectors (superotemporal, superior, superonasal, inferonasal, inferior, and inferotemporal) Ganglion Cell-Inner Plexiform Layer thickness (GCL + defined as the thickness from the inner boundary of the GCL to the outer boundary of the inner plexiform layer [IPL]). OCT scans were acquired by the same experienced operator (MC) and poor-quality images (quality index less than $60)$, segmentation or centered errors, presence of any optic disk abnormalities potentially interfering with the goodness of OCT examination (presence of dysmorphic or tilted optic disk) were rejected from OCT data analysis. Moreover, AD patients performed neuropsychological evaluation and the MMSE corrected (MMSEc score) was obtained for all of them. We also collected all the clinical information available, including concomitant medications potentially impacting on 
pupil function (Chougule et al., 2019; Kelbsch et al., 2019), for both controls and $\mathrm{AD}$ patients.

\section{Chromatic Pupillometric Protocol Apparatus, Stimuli, and Procedures}

A Ganzfeld ColorDome full-field stimulator (Espion V6, ColorDome Desktop Ganzfeld; Diagnosys LLC, Lowell, MA, United States) was used for the chromatic pupillometry test. Participants were dark-adapted for $10 \mathrm{~min}$ prior to start of the test. With the exclusion of patients for which only one eye was eligible for the study, we tested the dominant eye, and the contralateral eye was patched for monocular testing ( $65 \%$ of the tested eyes were right eyes). Colored light stimuli were presented to the tested eye and the pupil responses were recorded from the same eye using the Ganzfeld system equipped with an integrated pupillometer. The complete pupillometric protocol for isolating the rod-, mRGC-, and cone-contribution is described in details elsewhere (Park et al., 2011). For this study we considered the following conditions, as previously reported (Park et al., 2017):

1. Rod-condition: low luminance $\left(0.001 \mathrm{~cd} / \mathrm{m}^{2}\right)$ blue flash presented in the dark;

2. Melanopsin-condition: photopically-matched red and blue stimuli $\left(450 \mathrm{~cd} / \mathrm{m}^{2}\right)$ presented in the dark;

3. Cone-condition: red flash $\left(10 \mathrm{~cd} / \mathrm{m}^{2}\right)$ presented against the rod-suppressing blue adapting field $\left(6 \mathrm{~cd} / \mathrm{m}^{2}\right)$.

Stimuli consisted of short wavelength (blue, dominant wavelength of 460-485 $\mathrm{nm}$; mid $=472 \mathrm{~nm}$ ) and long wavelength (red, dominant wavelength of $620-645 \mathrm{~nm}$; mid $=632 \mathrm{~nm}$ ) light-flashes of $1 \mathrm{~s}$ duration. The integrated pupillometer system measured the pupil diameter at a $100 \mathrm{~Hz}$ sampling frequency. The interstimulus interval (ISI) was $20 \mathrm{~s}$ for the rod- and cone-conditions (for both red and blue stimuli), while for the melanopsin-condition ISI was $30 \mathrm{~s}$ for red stimulus and $70 \mathrm{~s}$ for the blue one. All recordings were completed in the same order with the red stimulus followed by the blue. For all three conditions, each stimulus was presented three times consecutively and the individual responses were obtained by their average recording. Participants were instructed to keep their eyes open during the duration of the light stimuli as well as following the stimuli. Participants who blinked frequently during the recordings were given another opportunity to repeat the measurements. Pupil traces with excessive artifacts due to long eye blinks or eye closure were excluded from subsequent pupillometric data analysis.

\section{Data Analysis}

Data were analyzed using custom scripts programmed in MATLAB (MathWorks Inc., Natick, MA, United States), which allowed for semi-automated analysis. PLR was normalized by the median steady-state (baseline) pupil size during the $2 \mathrm{~s}$ preceding each stimulus onset in order to minimize the effects of inter-subject differences in the baseline pupil size.

The following pupillometric parameters were calculated:

I. Transient PLR amplitude (or Transient Peak Amplitude) was defined as the difference between the normalized baseline and the minimum normalized PLR after stimulus onset (pupil maximum constriction);

II. For the melanopsin-condition, the PIPR was used for evaluating the mRGC sustained response. PIPR parameter was defined as the difference between the normalized baseline and the median normalized PLR measured over a 5 to $7 \mathrm{~s}$ time interval from stimulus offset. In particular, we evaluated PIPR from the blue and the red photopicallymatched stimuli, and also the difference between the blue PIPR and the red one $\left(\mathrm{PIPR}_{\text {Normalized }}=\mathrm{PIPR}_{\text {Blue }}\right.$ PIPR $_{\text {Red }}$ ).

\section{Statistical Analysis}

The Shapiro-Wilk and Kolmogorov-Smirnov tests were performed to assess the normal distribution and graphic inspection of the data. Chi-square and independent- $t$ tests were used to compare variables among groups. For continuous variables (pupillometric parameters), z-scores (standard scores) were also calculated. Levene's test was used to assess the equality of variances for mRGC sustained response for control and $\mathrm{AD}$ groups. Comparisons between groups for all pupillometric variables, measured under rod-, melanopsin-, and cone-mediated conditions, were computed by means of analysis of covariance (ANCOVA) with age as the covariate. Moreover, the $p$-value for interaction age $\times$ group was computed from the log-likelihood ratio test comparing ANCOVA models with and without the interaction term, and stratified $\beta$ coefficients (95\% Confidence Interval, $95 \% \mathrm{CI}$ ) for the variables turning out to be effect modifiers ( $p$-value for interaction $<0.15$ ) were presented.

For OCT data, we followed "one-eye" approach by evaluating the eye tested by chromatic pupillometry. Pearson correlation coefficients were used to measure the degree of association between pupillometric parameters and clinical data (OCT measures, MMSEc score, and disease duration) in control and Alzheimer's groups. Statistical analyses were performed using SPSS (SPSS Inc., IBM, Chicago, IL, United States) and Stata SE (StataCorp, College Station, TX, United States) softwares.

\section{RESULTS}

This study included 26 mild-moderate $\mathrm{AD}$ patients from 52 to 88 years of age (69.3 \pm 7 years) and 26 healthy participants (controls) from 58 to 82 years of age $(70.2 \pm 11$ years). The demographic and clinical data of the two groups are shown in Table 1. Controls and AD patients did not significantly differ in terms of age and gender (gender, $p=0.58$; age, $p=0.75$ ).

Raw pupil traces from two controls showed excessive blink artifacts under the rod- (blue flash) and melanopsin- (blue flash) conditions and were removed from data analysis. Further, four control and six $\mathrm{AD}$ pupil traces under the cone-condition showed too many artifacts, were not reliable, and thus were removed from data analysis.

The single normalized pupil traces (PLR curves) under all conditions are shown in Figure 1. For the rod- (Figures 1A,B) and cone- (Figures 1G,H) conditions, the PLR is characterized by a rapid transient constriction followed by a relatively rapid 
TABLE 1 | Sociodemographic data.

\begin{tabular}{lccc}
\hline & Controls & Alzheimer's & $\boldsymbol{p}$-value \\
\hline $\mathbf{N}$ & $26(50 \%)$ & $26(50 \%)$ & \\
Gender & $11(42.3 \%)$ & $14(53.8 \%)$ & 0.58 \\
Male & $15(57.7 \%)$ & $12(46.2 \%)$ & \\
Female & $69.3 \pm 7$ & $70.2 \pm 11$ & $0.75 \mid 0.16$ \\
Age | Age-class & $3(11.5 \%)$ & $6(23.1 \%)$ & \\
50-59 years & $10(38.5 \%)$ & $4(15.4 \%)$ & \\
60-69 years & $10(38.5 \%)$ & $9(34.6 \%)$ & \\
70-79 years & $3(11.5 \%)$ & $7(26.9 \%)$ & \\
80-89 years & $/$ & $20.7 \pm 4$ & \\
MMSEc & $/$ & $(17.5-24.8)$ & \\
& & $3.8 \pm 2.9$ & \\
Disease duration & & $(2-4.2)$ & \\
& & & \\
\hline
\end{tabular}

Values are given as $n$ (\%) or mean \pm standard deviation (interquartile range, Q1Q3). MMSEC, Mini Mental State Examination corrected score. Chi-square test was performed with categorical variables and independent-t test was performed with continuous variables.

return to the baseline both in controls (Figures $\mathbf{1 A , G}$ ) and in $\mathrm{AD}$ (Figures 1B,H).

Under the melanopsin-condition (blue flash), in both controls (Figure 1C) and AD (Figure 1D), the PLR is characterized by an initial transient constriction followed by a sustained constriction (PIPR) during the 5-7 s time interval from light-stimulus offset. Melanopsin-mediated sustained response is more variable in the $\mathrm{AD}$ group (Figure 1D) compared to the control group (Figure 1C). In fact, the estimated population variances of the PIPR at 5-s from stimulus offset of the two groups were statistically different (Levene's test: $\mathrm{SD}$ control $=0.03, \mathrm{SD} \mathrm{AD}=0.06 ; p=0.018)$ and, in particular, the variability in the $\mathrm{AD}$ group resulted significantly greater. Moreover, five AD patients showed a PIPR lower more than $2 \mathrm{SD}$ from the control mean value. Under the melanopsin-condition (red flash), in both controls (Figure 1E) and $\mathrm{AD}$ (Figure 1F), the elicited PLR is characterized by an initial transient constriction, followed by a smaller sustained response with a reduced amplitude in the $\mathrm{AD}$ group compared to controls.

The individual pupillometric parameters for controls and $\mathrm{AD}$ for the three conditions are provided in Figure 2. There was no difference between $\mathrm{AD}$ and control groups in terms of baseline normalized pupil size under any of the three conditions (Figures 2A,C,G and Supplementary Table 1). PLR transient amplitude (Figures 2B,E,F and Supplementary Table 1) was significantly decreased under rod- $(p=0.006)$ and melanopsin(blue flash, $p=0.02$; red flash, $p=0.006$ ) conditions in $\mathrm{AD}$ compared to controls. PIPR $\mathrm{Blue}_{\text {in }}$ in the melanopsin-condition was not significantly different between $\mathrm{AD}$ and controls (Figure 2D and Supplementary Table 1). PLR transient amplitude was not significantly different between $\mathrm{AD}$ and controls under the conecondition (Figure 2H and Supplementary Table $\mathbf{1}$ ).

Figure 3 shows the mean normalized pupil traces of rod- (Figure 3A), melanopsin- (Figure 3B), and cone(Figure 3C) conditions for each subject group. We failed to observe any difference in terms of $\mathrm{PIPR}_{\mathrm{Blue}}$ and $\mathrm{PIPR}_{\text {Normalized }}$ (Supplementary Table 1) between AD and controls.

To check the extent to which the control- and AD-regression lines of each pupillometric parameters with age deviate from parallel, the likelihood-ratio test was used (Supplementary Tables 2, 3 and Supplementary Figure 1). Likelihood-ratio test showed the existence of interaction age $\times$ group for

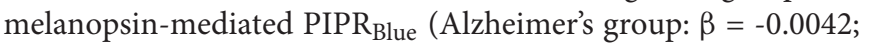
$95 \% \mathrm{CI}=-0.0073-0.0011 ; r=-0.5)$ and transient peak amplitude (Alzheimer's group, blue flash: $\beta=-0.0034 ; 95 \%$ $\mathrm{CI}=-0.0055-0.0014 ; r=-0.59$; red flash: $\beta=-0.0021 ; 95 \%$ $\mathrm{CI}=-0.0039-0.0003 ; r=-0.44)$ parameters with a significant correlation only in AD (Supplementary Tables 2, 3 and Supplementary Figures 1B,D,F).

Furthermore, Pearson's correlation analysis was used to determine if there was a relationship between the calculated pupillometric parameters and OCT measures, MMSEc score, and disease duration (the latter two only for AD). There was no significant correlation between pupillometric parameters and OCT measurements, neither with MMSEc score and disease duration for AD patients. Mean comparisons of OCT measurements for all RNFL quadrants (temporal, superior, nasal, and inferior) and macular GCL + sectors (superotemporal, superior, superonasal, inferonasal, inferior, and inferotemporal) did not show significant differences between $\mathrm{AD}$ and controls (data not shown).

We also retrieved information regarding oral medications that could potentially interfere with pupillary responses, i.e., cholinesterase inhibitors for $\mathrm{AD}$ and beta-blockers for controls. Only a few controls $(n=4,15.5 \%)$ were on beta-blockers (atenolol/metaprolol/bisoprolol), while $16 \mathrm{AD}(61.5 \%)$ were on cholinesterase inhibitors drugs (donepezil/rivastigmine/galantamine). In controls, there were no differences in terms of pupillometric parameters between subjects taking beta-blockers and those not taking them (data not shown). No significant differences among AD subgroups (cholinergic-treated and cholinergic-untreated) for any PLR parameters were found (data not shown).

\section{DISCUSSION}

In this paper, we evaluated for the first time, using chromatic pupillometry, aimed at isolating the mRGC contribution (Park et al., 2017), the PLR in a cohort of 26 definite mild-moderate $\mathrm{AD}$ patients compared to a group of age- and gender-matched controls. In particular, to specifically target mRGC function, we evaluated the PIPR amplitude using intense $\left(450 \mathrm{~cd} / \mathrm{m}^{2}\right)$ blue (472 nm) light stimuli. The PIPR, which is the most reliable marker of mRGC-mediated PLR, was not significantly different between $\mathrm{AD}$ and controls, but we found a significant difference in terms of transient PLR amplitude between AD and controls under dark-adaption. Concerning the PIPR, even though the difference between $\mathrm{AD}$ and controls was not significantly different, the $\mathrm{AD}$ group showed higher variability with five individuals having a $\mathrm{PIPR}_{\mathrm{Blue}}$ amplitude value outside the 2 $\mathrm{SD}$ range from the control mean. Such variability in terms of 


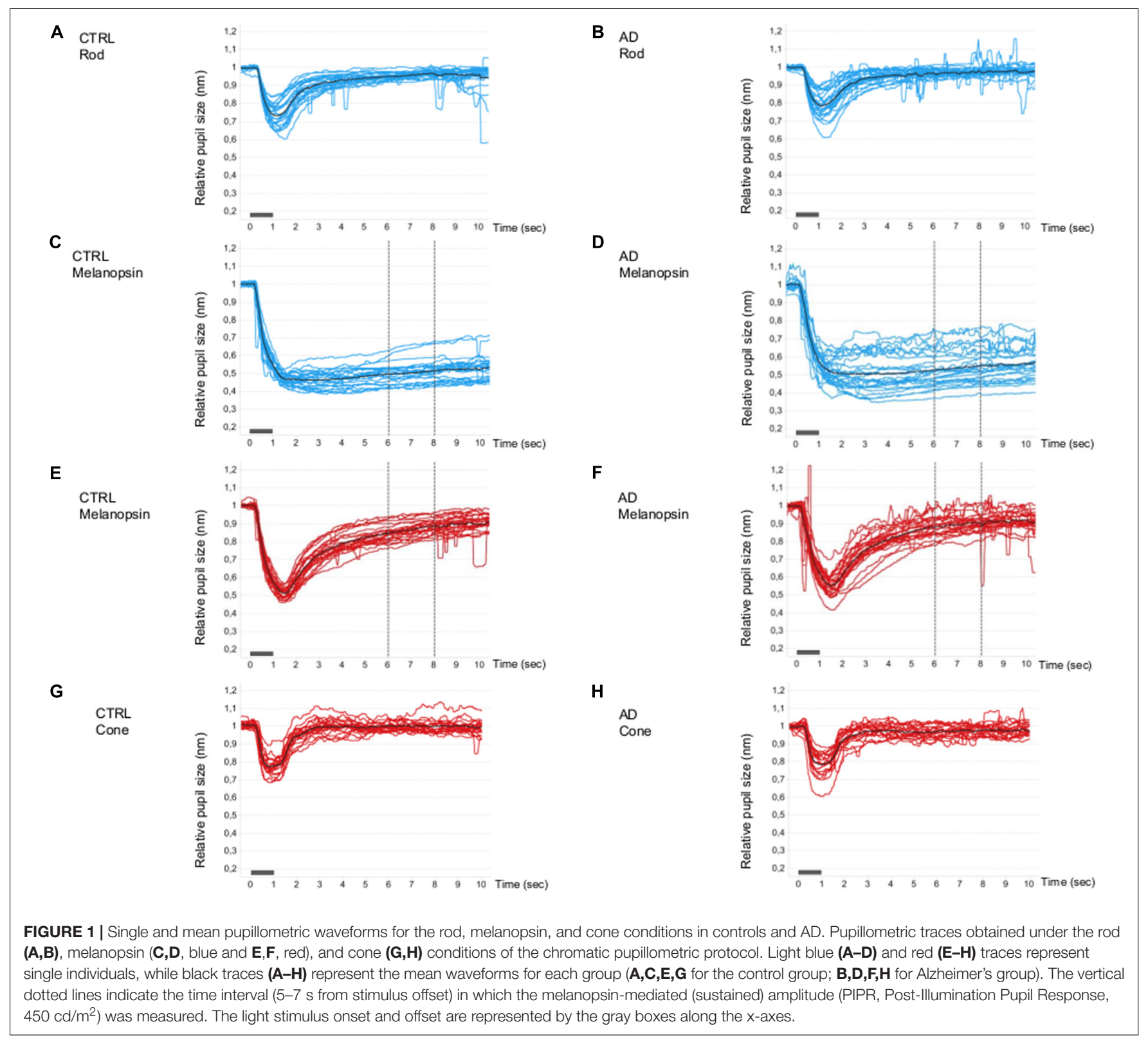

disease severity has been already reported in terms of circadian measurements and optic nerve pathology (La Morgia et al., 2016) and might depend on the severity as well as on disease duration (Hatfield et al., 2004). In this study we included AD patients in a mild-moderate stage of the disease and disease severity and duration were on average lower than previously published cohorts (La Morgia et al., 2016). In rodents, six different mRGC subtypes were characterized, and PLR was mainly regulated by the Brn3b-positive M1 and non-M1 subtypes (Chen et al., 2011; $\mathrm{Li}$ and Schmidt, 2018). In humans, Hannibal and colleagues also identified six subtypes of mRGCs (M1, M2, M3, M4, giant M1, and giant displaced M1), unevenly distributed across the human retina and with distinct anatomical characteristics (Hannibal et al., 2017). We previously demonstrated in post-mortem AD retinas that mRGCs are lost in $\mathrm{AD}$ and amyloid pathology specifically affects these cells (La Morgia et al., 2016). However, it is not known whether in $\mathrm{AD}$ the neurodegenerative process affects a specific mRGC subtype, and in particular those mRGC contributing to the PLR.

Interestingly, we found a significant difference in terms of transient peak amplitude both under the rod-condition, using the short duration, narrowband pulse, and low intensity $\left(0.001 \mathrm{~cd} / \mathrm{m}^{2}\right)$ blue light stimulus, and under the melanopsincondition, but not in the cone-condition, overall pointing to a prominent rod-mediated response (McDougal and Gamlin, 2010; Kostic et al., 2016; Krishnan et al., 2020). Considering that mRGCs receive synaptic input from rods and cones through bipolar cells and a direct contact of rod bipolar cells via ribbon synapses in the ON layer of the IPL with mRGCs has been demonstrated in human retinas (Hannibal et al., 2017), 


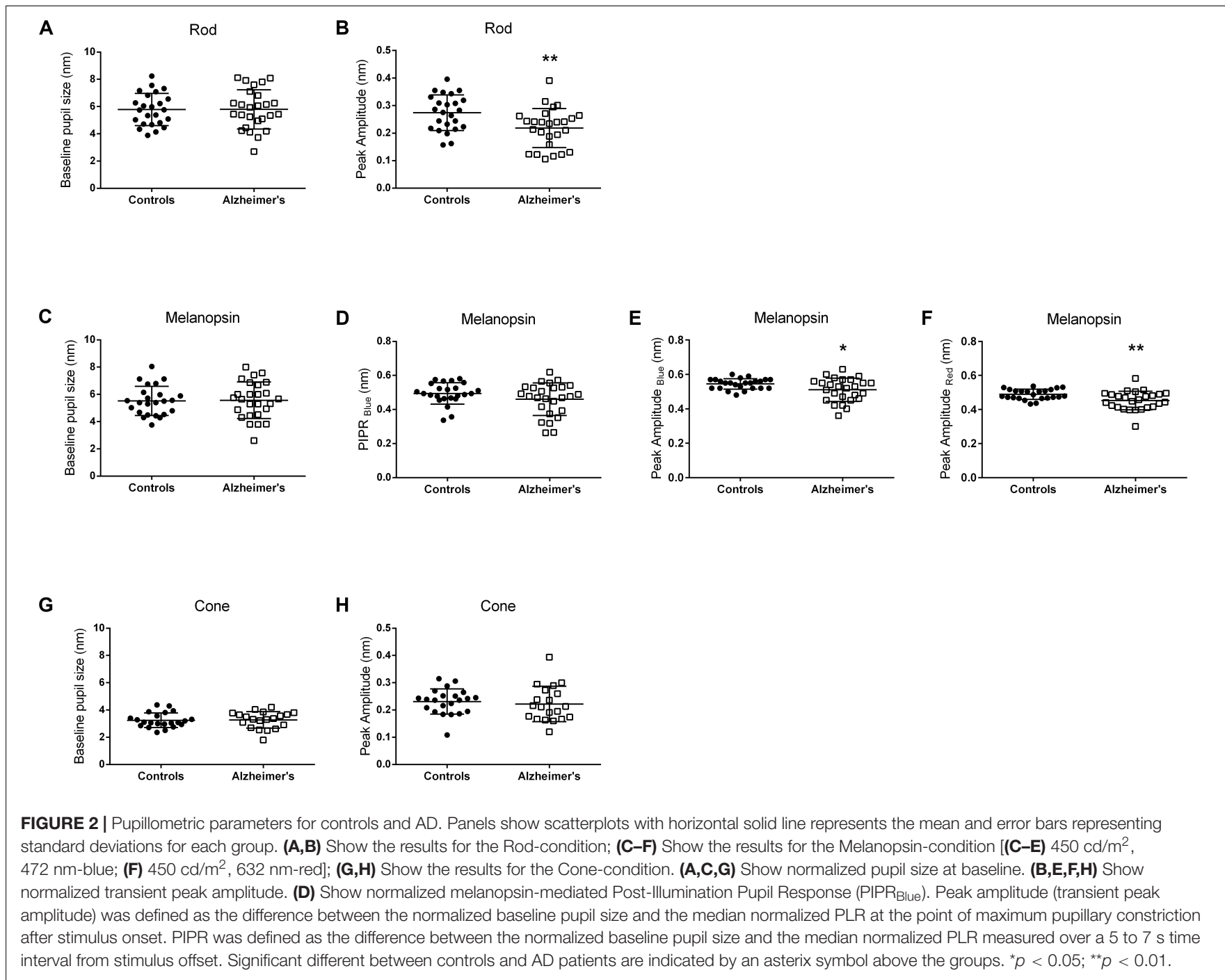

this difference between $\mathrm{AD}$ and controls could suggest that in the early stages of the disease there is no obvious cell body dysfunction but possibly a dendropathy. This suggestion is based on the presence of mRGC dendrite pathology, previously reported in $\mathrm{AD}$, with extensive morphological abnormalities in the spared mRGCs showing dendrite varicosities, patchy distribution of melanopsin, and reduced dendrite arborization (La Morgia et al., 2016). Dendritic degeneration has been also documented in RGCs of AD mouse model (Williams et al., 2013), and there are other disease models such as OPA1-related optic atrophy in which dendrites are the primary site of pathology (Williams et al., 2010). The different mRGC subtypes are distinctively connected to rods and cones and specifically modulated by various light conditions (Weng et al., 2013). Furthermore, the presence of a contact from amacrine cells and directly from rod bipolar cells via ribbon synapses on M1, M2, and M4 soma membrane and dendrites has been demonstrated in human retinas (Hannibal et al., 2017). The significantly reduced transient peak amplitude in conditions exploring the rod-contribution may thus suggest an altered contact between rods and mRGCs. It is possible to hypothesize that the rod response depends more on the distal dendrites, and consequently that the subsequent reduced dendritic arborization might interfere with the rod input out of proportion to the cones. However, we cannot exclude that the rod-mediated mRGC dysfunction in $\mathrm{AD}$ can be due to pathology specifically affecting rod-bipolar cells while possibly sparing cone-bipolar cells.

In summary, pathology often provokes compensation. This is particularly true with the central nervous system, which has many gain control circuits in place (Ostergaard et al., 2007; Do, 2019). Therefore, if disease, injury, or aging causes a reduction of units, there are many means for restoring the overall average mass effect. However, with coarser granularity, there is increased variability (Mendell, 2014). In this case, fewer mRGCs or even fewer dendritic circuits give less granularity in the system and a tendency for larger swings in the response. Thus, variability would precede decompensation into failure.

Baseline pupil size was not significantly different between $\mathrm{AD}$ and controls. It must be considered, however, that $16 / 26$ 
A 1,2

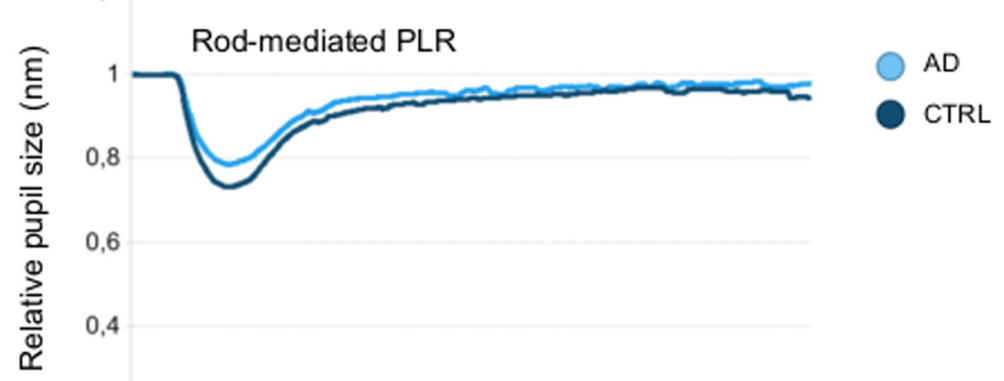

0,2

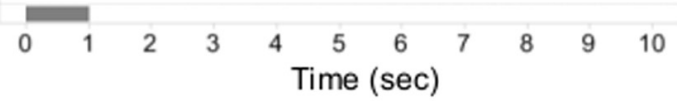

B

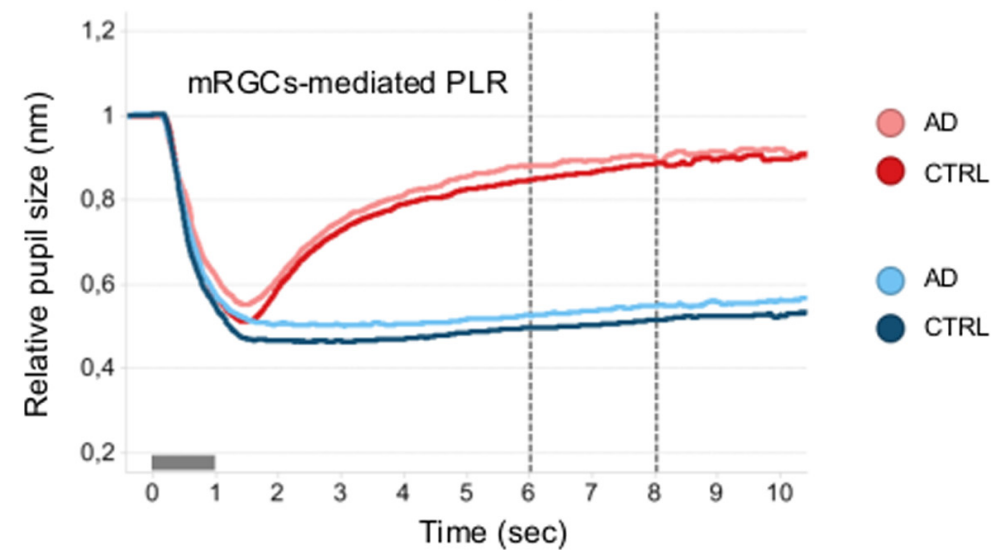

C

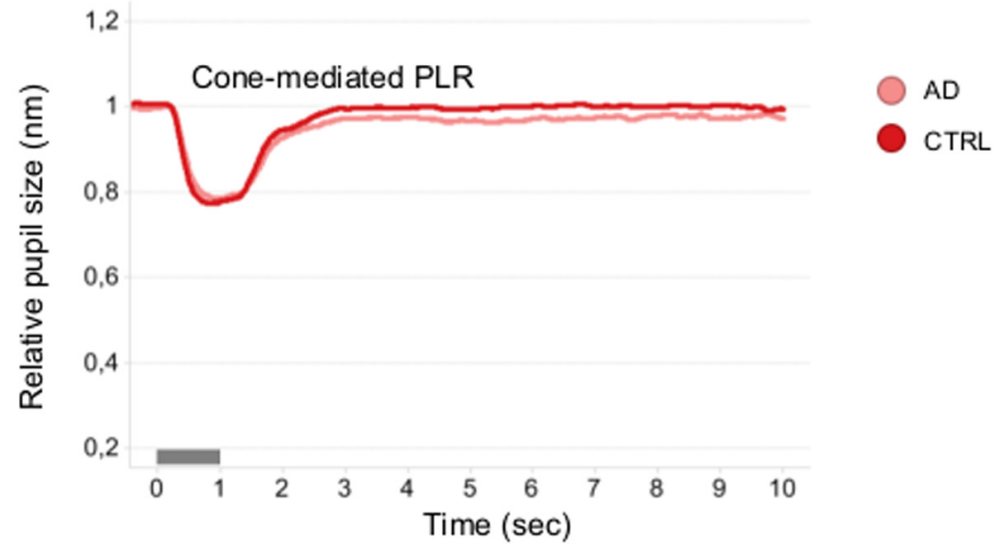

FIGURE 3 | Mean pupillometric waveforms obtained under the rod, melanopsin, and cone conditions in controls and AD. (A) Shows PLR measured under the rod-condition with the short- (blue) wavelength flashes $\left(0.001 \mathrm{~cd} / \mathrm{m}^{2}\right)$ presented in the dark, for comparison between control (blue mean trace) and AD (azure mean traces) groups. (B) Shows PLR measured under the melanopsin-condition, including the two photopically-matched intense long- (red) and short- (blue) wavelength flashes $\left(450 \mathrm{~cd} / \mathrm{m}^{2}\right)$ presented in the dark, for comparison between control (red and blue mean traces) and AD (pink and azure mean traces) groups. The vertical dotted lines indicate the time interval (5-7 s from stimulus offset) over which the melanopsin-mediated (sustained) amplitude (PIPR) was measured. (C) Shows PLR measured with the long- (red) wavelength flashes $\left(10 \mathrm{~cd} / \mathrm{m}^{2}\right)$ presented against the rod-suppressing blue adapting field $\left(6 \mathrm{~cd} / \mathrm{m}^{2}\right)$ for comparison between control (red mean trace) and $\mathrm{AD}$ (pink mean trace) groups.

$\mathrm{AD}$ patients were on acetylcholinesterase inhibitor drugs, and this might have an impact on the baseline pupil size. A few, small sample studies have reported the effect on the PLR of commonly used cholinergic AD drugs (Fotiou et al., 2000; Granholm et al., 2003). In one of these studies the authors did not find an effect of cholinergic medications on baseline pupil size, but demonstrated an increase in pupil constriction latency (Granholm et al., 2003). We also compared the PLR in AD patients taking and not taking these drugs and failed to demonstrate significant differences. Additional studies are needed to conclude a real effect of cholinergic medications on PLR and to clarify 
if different acetylcholinesterase inhibitors could have a different impact on PLR.

Previous studies investigated PLR in AD patients documenting reduced velocity, constriction amplitude, and increased latencies of PLR. These results were interpreted as related to the acetylcholine deficiency and parasympathetic dysfunction in AD (Prettyman et al., 1997; Fotiou et al., 2000, 2007, 2009; Tales et al., 2001; Granholm et al., 2003; Frost S. et al., 2013; Frost S.M. et al., 2013; Frost et al., 2017; Bittner et al., 2014; Chougule et al., 2019). Unfortunately, none of these studies were based on chromatic pupillometry protocol, and these results were not confirmed by more recent studies, which focused on early and pre-clinical stages of AD (Chougule et al., 2019). One chromatic pupillometry study evaluated pre-symptomatic AD cases (Oh et al., 2019). Oh and co-authors evaluated the PLR response using a similar pupillometric protocol in a cohort of 10 pre-symptomatic $\mathrm{AD}$ cases, defined on the basis of the cerebrospinal fluid markers, and they did not demonstrate a significant difference between pre-symptomatic AD cases and controls (Oh et al., 2019). However, congruent to the current findings, higher variability of PLR was documented in the AD group (Oh et al., 2019). Moreover, in this study the authors used only the $2.3 \log \mathrm{cd} / \mathrm{m}^{2}$ photopically-matched red and blue stimuli (Oh et al., 2019). Similarly, Van Stavern and co-authors, using a white light stimulus, did not show any difference between preclinical AD subjects (defined by CSF markers) and normal aging controls in any of the PLR parameters examined (Van Stavern et al., 2019). Very recently, Kawasaki et al. (2020), using a different chromatic pupillometry protocol under photopic conditions, failed to demonstrate, similarly to our results, a significant difference between early $\mathrm{AD}$ and controls in terms of PIPR response.

We also demonstrated a significant correlation of the PIPR amplitude and transient peak amplitude (melanopsin-condition, $450 \mathrm{~cd} / \mathrm{m}^{2}$ ) with age only in the AD group, which is in line with previous results pointing to an accelerated aging process in AD (La Morgia et al., 2016). Data on PLR in relation to age are not conclusive even though the majority of papers failed to reveal a significant difference of PLR in relation to the aging process. However, the controls included in these studies were younger than 70 , and this might explain the absence of significant impairment of the pupil response (Adhikari et al., 2015a; Rukmini et al., 2017). Our results are in line with the observation of mRGC loss with age (Semo et al., 2003; La Morgia et al., 2016; Esquiva et al., 2017).

We did not find a correlation between pupil metrics and OCT parameters nor with disease severity or duration. Moreover, we did not find any significant difference in terms of RNFL and GCL + thickness between AD and controls. Any effect specific to mRGC loss would have been swamped by regular RGCs in these measures. Further, these results can be explained by the inclusion of milder cases with shorter disease duration. A recent SS-OCT study evaluating a large cohort of AD cases failed to demonstrate a significant difference in terms of RNFL between $\mathrm{AD}$ and controls (Sanchez et al., 2018).

Overall, the current chromatic pupillometry findings in a cohort of mild-moderate AD patients did not demonstrate a clear
mRGC-driven pupil dysfunction but are rather consistent with a dendropathy in the early stage of the disease, supported by our previous post-mortem studies of $\mathrm{AD}$ retinas. Early pathology, while still in the range of compensatory mechanisms, often manifests as variability. Further studies including more severe and with longer disease duration $\mathrm{AD}$ cases are needed to further explore this hypothesis. Such studies may also clarify whether the PLR can be used as a tool evaluating the progression of the disease and eventually the efficacy of therapies in AD.

\section{DATA AVAILABILITY STATEMENT}

The raw data supporting the conclusion of this article will be made available from the corresponding author, without undue reservation, to any qualified researcher.

\section{ETHICS STATEMENT}

The studies involving human participants were reviewed and approved by the EC Interaziendale Bologna-Imola \#16032. The patients/participants provided their written informed consent to participate in this study.

\section{AUTHOR CONTRIBUTIONS}

MR: acquisition, analysis and interpretation of data, and drafting and revising the work. MS, MD, SC, MC, GA, and CL: data acquisition and revising the work. $\mathrm{MR}$ and $\mathrm{CZ}$ : statistical data analysis. MR, MC, GC, RL, AS, VC, JP, and CL: contributed to interpretation of the data. MR, GC, VC, JP, and CL: design of the work. MS, MD, SC, MC, GA, GC, CZ, RL, AS, VC, JP, and CL: revising the work and provided approval for publication of the content. All authors contributed to the article and approved the submitted version.

\section{FUNDING}

This work was supported by the Italian Ministry of Health Young Researcher Project Grant (GR-2013-02358026 to CL).

\section{ACKNOWLEDGMENTS}

We were deeply grateful to patients and their caregivers for participating to the study. We also thank Prof. Parchi for performing the CSF examination of the AD subjects included and Dr. Vincenzo Allegri for performing amyloid PET in AD patients. We also thank Luca Berti for his assistance in pupil traces analysis and Prof. Jens Hannibal for his advices.

\section{SUPPLEMENTARY MATERIAL}

The Supplementary Material for this article can be found online at: https://www.frontiersin.org/articles/10.3389/fnins.2020. 00780/full\#supplementary-material 


\section{REFERENCES}

Adhikari, P., Pearson, C. A., Anderson, A. M., Zele, A. J., and Feigl, B. (2015a). Effect of age and refractive error on the melanopsin mediated post-illumination pupil response (PIPR). Sci. Rep. 5:17610. doi: 10.1038/srep17610

Adhikari, P., Zele, A. J., and Feigl, B. (2015b). The post-Illumination pupil response (PIPR). Invest. Ophthalmol. Vis. Sci. 56, 3838-3849. doi: 10.1167/iovs.14-16233

Baver, S. B., Pickard, G. E., Sollars, P. J., and Pickard, G. E. (2008). Two types of melanopsin retinal ganglion cell differentially innervate the hypothalamic suprachiasmatic nucleus and the olivary pretectal nucleus. Eur. J. Neurosci. 27, 1763-1770. doi: 10.1111/j.1460-9568.2008.06149.x

Beck, A. T., Epstein, N., Brown, G., and Steer, R. A. (1988). An inventory for measuring clinical anxiety: psychometric properties. J. Consult. Clin. Psychol. 56, 893-897. doi: 10.1037//0022-006x.56.6.893

Beck, A. T., Ward, C. H., Mendelson, M., Mock, J., and Erbaugh, J. (1961). An inventory for measuring depression. Arch. Gen. Psychiatry 4, 561-571. doi: 10.1001/archpsyc. 1961.01710120031004

Berson, D. M., Dunn, F. A., and Takao, M. (2002). Phototransduction by retinal ganglion cells that set the circadian clock. Science 295, 1070-1073. doi: 10.1126/ science.1067262

Bittner, D. M., Wieseler, I., Wilhelm, H., Riepe, M. W., and Muller, N. G. (2014). Repetitive pupil light reflex: potential marker in Alzheimer's disease? J. Alzheimers Dis. 42, 1469-1477. doi: 10.3233/JAD-140969

Buysse, D. J., Reynolds, C. F. III, Monk, T. H., Berman, S. R., and Kupfer, D. J. (1989). The Pittsburgh sleep quality index: a new instrument for psychiatric practice and research. Psychiatry Res. 28, 193-213. doi: 10.1016/0165-1781(89) 90047-4

Chen, S. K., Badea, T. C., and Hattar, S. (2011). Photoentrainment and pupillary light reflex are mediated by distinct populations of ipRGCs. Nature 476, 92-95. doi: 10.1038/nature10206

Chougule, P. S., Najjar, R. P., Finkelstein, M. T., Kandiah, N., and Milea, D. (2019). Light-induced pupillary responses in Alzheimer's Disease. Front .Neurol. 10:360. doi: 10.3389/fneur.2019.00360

Do, M. T. H. (2019). Melanopsin and the intrinsically photosensitive retinal ganglion cells: biophysics to behavior. Neuron 104, 205-226. doi: 10.1016/j. neuron.2019.07.016

Dubois, B., Feldman, H. H., Jacova, C., Hampel, H., Molinuevo, J. L., Blennow, K., et al. (2014). Advancing research diagnostic criteria for Alzheimer's disease: the IWG-2 criteria. Lancet Neurol. 13, 614-629. doi: 10.1016/S1474-4422(14) 70090-0

Esquiva, G., Lax, P., Perez-Santonja, J. J., Garcia-Fernandez, J. M., and Cuenca, N. (2017). Loss of melanopsin-expressing ganglion cell subtypes and dendritic degeneration in the aging human retina. Front. Aging Neurosci. 9:79. doi: 10. 3389/fnagi.2017.00079

Folstein, M. F., Folstein, S. E., and McHugh, P. R. (1975). "Mini-mental state". A practical method for grading the cognitive state of patients for the clinician. J. Psychiatr. Res. 12, 189-198. doi: 10.1016/0022-3956(75)90026-6

Fotiou, D. F., Brozou, C. G., Haidich, A. B., Tsiptsios, D., Nakou, M., Kabitsi, A., et al. (2007). Pupil reaction to light in Alzheimer's disease: evaluation of pupil size changes and mobility. Aging Clin. Exp. Res. 19, 364-371. doi: 10.1007/ bf03324716

Fotiou, D. F., Stergiou, V., Tsiptsios, D., Lithari, C., Nakou, M., and Karlovasitou, A. (2009). Cholinergic deficiency in Alzheimer's and Parkinson's disease: evaluation with pupillometry. Int. J. Psychophysiol. 73, 143-149. doi: 10.1016/ j.ijpsycho.2009.01.011

Fotiou, F., Fountoulakis, K. N., Tsolaki, M., Goulas, A., and Palikaras, A. (2000). Changes in pupil reaction to light in Alzheimer's disease patients: a preliminary report. Int. J. Psychophysiol. 37, 111-120. doi: 10.1016/s0167-8760(00)00099-4

Frost, S., Kanagasingam, Y., Sohrabi, H., Bourgeat, P., Villemagne, V., Rowe, C. C., et al. (2013). Pupil response biomarkers for early detection and monitoring of Alzheimer's disease. Curr. Alzheimer Res. 10, 931-939. doi: 10. 2174/15672050113106660163

Frost, S. M., Kanagasingam, Y., Sohrabi, H. R., Taddei, K., Bateman, R., Morris, J., et al. (2013). Pupil response biomarkers distinguish amyloid precursor protein mutation carriers from non-carriers. Curr. Alzheimer Res. 10, 790-796. doi: $10.2174 / 15672050113109990154$

Frost, S., Robinson, L., Rowe, C. C., Ames, D., Masters, C. L., Taddei, K., et al. (2017). Evaluation of cholinergic deficiency in preclinical Alzheimer's Disease using pupillometry. J. Ophthalmol. 2017:7935406. doi: 10.1155/2017/793 5406

Granholm, E., Morris, S., Galasko, D., Shults, C., Rogers, E., and Vukov, B. (2003). Tropicamide effects on pupil size and pupillary light reflexes in Alzheimer's and Parkinson's disease. Int. J. Psychophysiol. 47, 95-115. doi: 10.1016/s01678760(02)00122-8

Hannibal, J., Christiansen, A. T., Heegaard, S., Fahrenkrug, J., and Kiilgaard, J. F. (2017). Melanopsin expressing human retinal ganglion cells: subtypes, distribution, and intraretinal connectivity. J. Comp. Neurol. 525, 1934-1961. doi: 10.1002/cne.24181

Hannibal, J., Hindersson, P., Knudsen, S. M., Georg, B., and Fahrenkrug, J. (2002). The photopigment melanopsin is exclusively present in pituitary adenylate cyclase-activating polypeptide-containing retinal ganglion cells of the retinohypothalamic tract. J. Neurosci. 22:RC191.

Hannibal, J., Hindersson, P., Ostergaard, J., Georg, B., Heegaard, S., Larsen, P. J., et al. (2004). Melanopsin is expressed in PACAP-containing retinal ganglion cells of the human retinohypothalamic tract. Invest. Ophthalmol. Vis. Sci. 45, 4202-4209. doi: 10.1167/iovs.04-0313

Hannibal, J., Kankipati, L., Strang, C. E., Peterson, B. B., Dacey, D., and Gamlin, P. D. (2014). Central projections of intrinsically photosensitive retinal ganglion cells in the macaque monkey. J. Comp. Neurol. 522, 2231-2248. doi: 10.1002/ cne. 23588

Hatfield, C. F., Herbert, J., van Someren, E. J., Hodges, J. R., and Hastings, M. H. (2004). Disrupted daily activity/rest cycles in relation to daily cortisol rhythms of home-dwelling patients with early Alzheimer's dementia. Brain 127(Pt 5), 1061-1074. doi: 10.1093/brain/awh129

Hattar, S., Liao, H. W., Takao, M., Berson, D. M., and Yau, K. W. (2002). Melanopsin-containing retinal ganglion cells: architecture, projections, and intrinsic photosensitivity. Science 295, 1065-1070. doi: 10.1126/science.106 9609

Kardon, R., Anderson, S. C., Damariian, T. G., Grace, E. M., Stone, E., and Kawasaki, A. (2011). Chromatic pupillometry in patients with retinitis pigmentosa. Ophthalmology 118, 376-381. doi: 10.1016/j.ophtha.2010.06.033

Kawasaki, A., Ouanes, S., Crippa, S. V., and Popp, J. (2020). Early-stage Alzheimer's disease does not alter pupil responses to colored light stimuli. J. Alzheimers Dis. 75, 1273-1282. doi: 10.3233/JAD-200120

Kelbsch, C., Strasser, T., Chen, Y., Feigl, B., Gamlin, P. D., Kardon, R., et al. (2019). Standards in pupillography. Front. Neurol. 10:129. doi: 10.3389/fneur. 2019.00129

Koronyo, Y., Biggs, D., Barron, E., Boyer, D. S., Pearlman, J. A., Au, W. J., et al. (2017). Retinal amyloid pathology and proof-of-concept imaging trial in Alzheimer's disease. JCI Insight 2:e93621. doi: 10.1172/jci.insight.93621

Kostic, C., Crippa, S. V., Martin, C., Kardon, R. H., Biel, M., Arsenijevic, Y., et al. (2016). Determination of rod and cone influence to the early and late dynamic of the pupillary light response. Invest. Ophthalmol. Vis. Sci. 57, 2501-2508. doi: 10.1167/iovs.16-19150

Krishnan, A. K., Jacobson, S. G., Roman, A. J., Iyer, B. S., Garafalo, A. V., Heon, E., et al. (2020). Transient pupillary light reflex in CEP290- or NPHP5-associated Leber congenital amaurosis: latency as a potential outcome measure of cone function. Vis. Res. 168, 53-63. doi: 10.1016/j.visres.2020.01.006

La Morgia, C., Carelli, V., and Carbonelli, M. (2018). Melanopsin retinal ganglion cells and pupil: clinical implications for neuro-ophthalmology. Front. Neurol. 9:1047. doi: 10.3389/fneur.2018.01047

La Morgia, C., Ross-Cisneros, F. N., Koronyo, Y., Hannibal, J., Gallassi, R., Cantalupo, G., et al. (2016). Melanopsin retinal ganglion cell loss in Alzheimer disease. Ann. Neurol. 79, 90-109. doi: 10.1002/ana.24548

La Morgia, C., Ross-Cisneros, F. N., Sadun, A. A., and Carelli, V. (2017). Retinal ganglion cells and circadian rhythms in Alzheimer's Disease. Parkinson's Disease, and Beyond. Front. Neurol. 8:162. doi: 10.3389/fneur.2017.00162

Li, J. Y., and Schmidt, T. M. (2018). Divergent projection patterns of M1 ipRGC subtypes. J. Comp. Neurol. 526, 2010-2018. doi: 10.1002/cne.24469

McDougal, D. H., and Gamlin, P. D. (2010). The influence of intrinsicallyphotosensitive retinal ganglion cells on the spectral sensitivity and response dynamics of the human pupillary light reflex. Vis. Res. 50, 72-87. doi: 10.1016/j. visres.2009.10.012

McKhann, G., Drachman, D., Folstein, M., Katzman, R., Price, D., and Stadlan, E. M. (1984). Clinical diagnosis of Alzheimer's disease: report of the NINCDSADRDA Work Group under the auspices of Department of Health and Human 
Services Task Force on Alzheimer's Disease. Neurology 34, 939-944. doi: 10. 1212/wnl.34.7.939

Mendell, L. M. (2014). Constructing and deconstructing the gate theory of pain. Pain 155, 210-216. doi: 10.1016/j.pain.2013.12.010

Oh, A. J., Amore, G., Sultan, W., Asanad, S., Park, J. C., Romagnoli, M., et al. (2019). Pupillometry evaluation of melanopsin retinal ganglion cell function and sleepwake activity in pre-symptomatic Alzheimer's disease. PLoS One 14:e0226197. doi: 10.1371/journal.pone.0226197

Ostergaard, J., Hannibal, J., and Fahrenkrug, J. (2007). Synaptic contact between melanopsin-containing retinal ganglion cells and rod bipolar cells. Invest. Ophthalmol. Vis. Sci. 48, 3812-3820. doi: 10.1167/iovs.06-1322

Park, J. C., Chen, Y. F., Blair, N. P., Chau, F. Y., Lim, J. I., Leiderman, Y. I., et al. (2017). Pupillary responses in non-proliferative diabetic retinopathy. Sci. Rep. 7:44987. doi: 10.1038/srep44987

Park, J. C., Moura, A. L., Raza, A. S., Rhee, D. W., Kardon, R. H., and Hood, D. C. (2011). Toward a clinical protocol for assessing rod, cone, and melanopsin contributions to the human pupil response. Invest. Ophthalmol. Vis. Sci. 52, 6624-6635. doi: 10.1167/iovs.11-7586

Prettyman, R., Bitsios, P., and Szabadi, E. (1997). Altered pupillary size and darkness and light reflexes in Alzheimer's disease. J. Neurol. Neurosurg. Psychiatry 62, 665-668. doi: 10.1136/jnnp.62.6.665

Rukmini, A. V., Milea, D., Aung, T., and Gooley, J. J. (2017). Pupillary responses to short-wavelength light are preserved in aging. Sci. Rep. 7:43832. doi: 10.1038/ srep43832

Sadun, A. A., Schaechter, J. D., and Smith, L. E. (1984). A retinohypothalamic pathway in man: light mediation of circadian rhythms. Brain Res. 302, 371-377. doi: 10.1016/0006-8993(84)90252-x

Sanchez, D., Castilla-Marti, M., Rodriguez-Gomez, O., Valero, S., Piferrer, A., Martinez, G., et al. (2018). Usefulness of peripapillary nerve fiber layer thickness assessed by optical coherence tomography as a biomarker for Alzheimer's disease. Sci. Rep. 8:16345. doi: 10.1038/s41598-018-34577-3

Semo, M., Lupi, D., Peirson, S. N., Butler, J. N., and Foster, R. G. (2003). Light-induced $\mathrm{c}$-fos in melanopsin retinal ganglion cells of young and aged rodless/coneless (rd/rd cl) mice. Eur. J. Neurosci. 18, 3007-3017. doi: 10.1111/j. 1460-9568.2003.03061.x

Tales, A., Troscianko, T., Lush, D., Haworth, J., Wilcock, G. K., and Butler, S. R. (2001). The pupillary light reflex in aging and Alzheimer's disease. Aging 13, 473-478.
Uddin, M. S., Tewari, D., Mamun, A. A., Kabir, M. T., Niaz, K., Wahed, M. I. I., et al. (2020). Circadian and sleep dysfunction in Alzheimer's Disease. Ageing Res. Rev. 60:101046. doi: 10.1016/j.arr.2020.101046

Van Stavern, G. P., Bei, L., Shui, Y. B., Huecker, J., and Gordon, M. (2019). Pupillary light reaction in preclinical Alzheimer's disease subjects compared with normal ageing controls. Br. J. Ophthalmol. 103, 971-975. doi: 10.1136/bjophthalmol2018-312425

Vignatelli, L., Plazzi, G., Barbato, A., Ferini-Strambi, L., Manni, R., Pompei, F., et al. (2003). Italian version of the Epworth sleepiness scale: external validity. Neurol. Sci. 23, 295-300. doi: 10.1007/s100720300004

von Elm, E., Altman, D. G., Egger, M., Pocock, S. J., Gotzsche, P. C., Vandenbroucke, J. P., et al. (2007). The strengthening the reporting of observational studies in epidemiology (STROBE) statement: guidelines for reporting observational studies. Lancet 370, 1453-1457. doi: 10.1016/S01406736(07)61602-X

Weng, S., Estevez, M. E., and Berson, D. M. (2013). Mouse ganglion-cell photoreceptors are driven by the most sensitive rod pathway and by both types of cones. PLoS One 8:e66480. doi: 10.1371/journal.pone.0066480

Williams, P. A., Morgan, J. E., and Votruba, M. (2010). Opal deficiency in a mouse model of dominant optic atrophy leads to retinal ganglion cell dendropathy. Brain 133, 2942-2951. doi: 10.1093/brain/awq218

Williams, P. A., Thirgood, R. A., Oliphant, H., Frizzati, A., Littlewood, E., Votruba, M., et al. (2013). Retinal ganglion cell dendritic degeneration in a mouse model of Alzheimer's disease. Neurobiol. Aging 34, 1799-1806. doi: 10.1016/j. neurobiolaging.2013.01.006

Conflict of Interest: The authors declare that the research was conducted in the absence of any commercial or financial relationships that could be construed as a potential conflict of interest.

Copyright (C) 2020 Romagnoli, Stanzani Maserati, De Matteis, Capellari, Carbonelli, Amore, Cantalupo, Zenesini, Liguori, Sadun, Carelli, Park and La Morgia. This is an open-access article distributed under the terms of the Creative Commons Attribution License (CC BY). The use, distribution or reproduction in other forums is permitted, provided the original author(s) and the copyright owner(s) are credited and that the original publication in this journal is cited, in accordance with accepted academic practice. No use, distribution or reproduction is permitted which does not comply with these terms. 